\title{
Interpretation of the pressuremeter test using numerical models based on deformation tensor equations
}

\author{
F. Escolano Sánchez $\cdot$ M. Bueno Aguado • \\ José Ramón Sánchez Lavín
}

\begin{abstract}
The pressuremeter test in boreholes has proven itself as a useful tool in geotechnical explorations, especially comparing its results with those obtained from a mathematical model ruled by a soil representative constitutive equation. The numerical model shown in this paper is aimed to be the reference framework for the interpretation of this test. The model analyses variables such as: the type of response, the initial state, the drainage regime and the constitutive equations. It is a model of finite elements able to work with a mesh without deformation or one adapted to it.
\end{abstract}

Keywords Pressuremeter tests - Constitutive equations $\cdot$ Geotechnical parameters $\cdot$ Finite elements

\section{Concept and introduction}

As it is well known, the pressuremeter test is carried out inside the boreholes (AENOR 1999). It is an in situ test in which lateral pressures are exerted on the soil through a central cell. In that way, the successive soil deformations in contact with the pressuremeter cell are registered at constant intervals of time and after having reached the pressure-deformation corresponding level (Yagiz et al. 2008).

The pressuremeter tests reduce mostly the disturbance of the soil mechanical features, which occurs during the samples taking. It also makes it possible to test the soil in a similar state to its natural one of effective pore pressures. On the other hand, the soil response is performed affecting a higher volume of material than the one tested in a laboratory, which makes it to come closer to the loading states that are used in engineering works afterwards (Morilla 2012).

Therefore, it fulfils enough requirements to deserve some effort at numerical modelling, which allows for obtaining more accurate results about the initial conditions of the soil and the deformation tensor that occurs around the cell (Haberfield and Johnston 1990; Clough 1990).

This paper depicts a numerical modelling based on the finite element method, purposely designed for this test, which allows for analysing a high amount of variables that influence the shape of curves resulting from the test. The comparison of these curves with the numerical reference framework implies an approach to the soil response and a method to determine its geomechanical parameters.

The numerical model and the results obtained modifying different variables are detailed in the following sections. Among them, the constitutive equations employed are the basis to obtain the geomechanical parameters and, consequently, to achieve a complete geotechnical diagnosis of the soil.

\section{Numerical modelling of the soil}

The modelling of the soil is carried out, in this analysis, through a discretization in concentric rings or spheres. Figure 1 shows a schematic representation of the mode of analysis. 


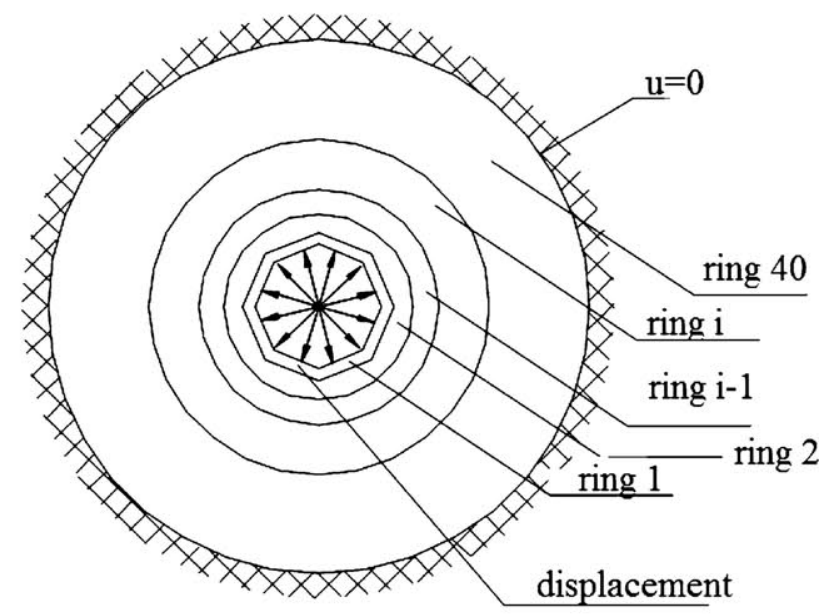

Fig. 1 Concentric rings or spheres model

It is a three-dimensional model with only one degree of freedom, whose variable is the displacement of the different ring or sphere edges.

The deformations obtained are the result of three possible kinds of response: no vertical deformation, constant vertical tension or spherical deformation.

The total external radius of the model is fixed, so that the magnitude of the external ring deformation is inferior to the one of the inner ring. Therefore, the deformations beyond the external ring influence negligibly on the movement calculated for the rings next to the cavity.

The total radial pressure of the first ring is the same as the pressure exerted inside the borehole. The condition of invalid movement is established on the external edge of the last ring.

The model is solved by the finite elements method. For each element " $i$ " the area of movement is represented by the function:

$u=N_{1}^{i} u_{i}+N_{2}^{i} u_{i+1}$,

$N_{1}^{i}=\frac{r_{2}-r}{r_{2}-r_{1}}$,

$N_{2}^{i}=\frac{r-r_{1}}{r_{2}-r_{1}}$.

From the area of movement the increments of deformation are obtained by the expression:

$$
\left(\begin{array}{c}
\Delta \varepsilon_{r} \\
\Delta \varepsilon_{\theta} \\
\Delta \varepsilon_{\gamma}
\end{array}\right)=\left(\begin{array}{cc}
\frac{-1}{r_{i+1}-r_{i}} & \frac{1}{r_{i+1}-r_{i}} \\
\frac{N_{1}^{i}}{r} & \frac{N_{2}^{i}}{r} \\
\frac{N_{1}^{i}}{r} & \frac{N_{2}^{i}}{r}
\end{array}\right)\left(\begin{array}{c}
u_{i} \\
u_{i+1}
\end{array}\right) .
$$

Spherical model

$$
\left(\begin{array}{c}
\Delta \varepsilon_{r} \\
\Delta \varepsilon_{\theta}
\end{array}\right)=\left(\begin{array}{cc}
\frac{-1}{r_{i+1}-r_{i}} & \frac{1}{r_{i+1}-r_{i}} \\
\frac{N_{1}^{i}}{r} & \frac{N_{2}^{i}}{r}
\end{array}\right)\left(\begin{array}{c}
u_{i} \\
u_{i+1}
\end{array}\right)
$$

\section{Axisymmetric model}

The stiffness matrix is obtained from the theorem of the virtual works, through the expression:

$\int_{\Omega} \delta \varepsilon \Delta \sigma \mathrm{d} \Omega=P_{1} \Gamma$,

in which $\Omega$ is the volume of soil tested, $\Gamma$ the inner area of the borehole and $P_{1}$ the increment of pressure exerted on the first ring.

The model is incrementally solved. An increase of pressure is exerted on the inner ring, and the movements are determined, with the deformation increments and through the constitutive equation, and the tension increments. The tension increments are added to the previous steps' tensions, the matrix of rigidity is modified and the following cycle begins.

\section{Types of response}

In the model considered there are three possible kinds of soil response (Arrua and Aiassa 2009):

1. Axisymmetric model with constant vertical pressure.

2. Axisymmetric model with invalid vertical deformation.

3. Spherical model.

The first kind of response is typical of tests carried out close to the surface where the vertical pressure is relatively low and its increase is impossible.

The model with invalid vertical deformation is illustrative of tests at a certain depth, where the vertical pressure increases and reduces significantly the vertical deformation.

The spherical model is a special case in which the two deformations are equal. The applied inner pressure is higher than in other kinds of response; so it could be concluded that it is a very deep test.

Three curves with the same geotechnical parameters and initial pressures, in which only the type of response has been modified, are shown in the following graph (Fig. 2). The corresponding constitutive equation is a perfect elastoplastic model.

It is observed that, according to the kind of response, the inner pressure for the same level of deformation is different.

Regarding to the elastic stretch, both axisymmetric kinds show the same behaviour in comparison to the spherical model where the inclination of the straight elastic stretch is higher.

Besides, only in the axisymmetric model with an invalid increment of vertical pressure, the inner pressure reaches a horizontal asymptote or limit pressure. In the other two kinds of response, the inner pressure always increases with deformation. 


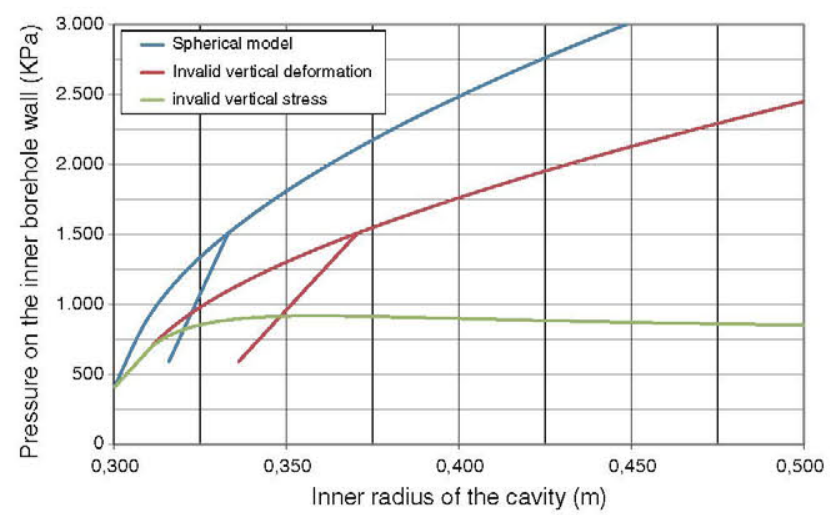

Fig. 2 Kinds of response

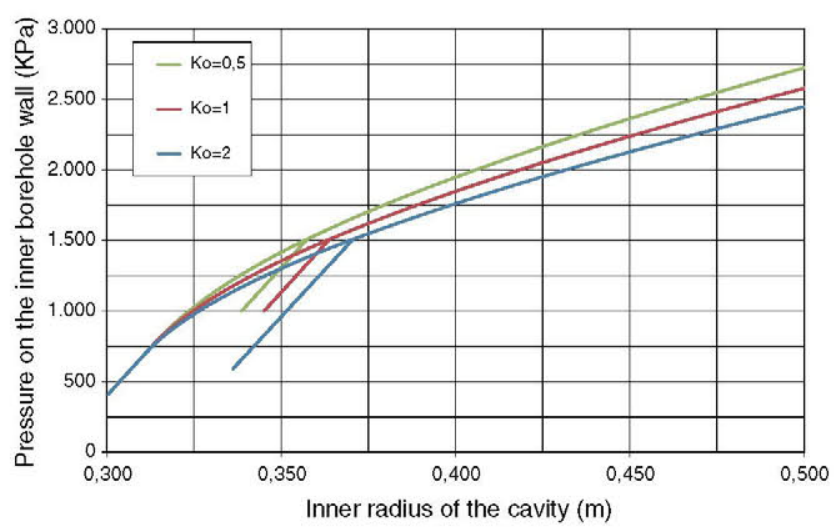

Fig. 3 Initial state

\section{Initial state}

The model requires the definition of an initial state of pressure. This state should coincide with the correspondent to the geostatic condition.

In the spherical model the initial state is defined by only one pressure which is equal in the three directions of space.

In the axisymmetric kind of response, the relation between the vertical pressure and the horizontal pressure (Ko parameters) can influence the response of the model. Three possible Ko values are analysed: 0.5, 1.0 and 2.0.

The inner pressure supported by the same levels of deformation, with only the coefficient Ko varying is shown in Fig. 3.

As the vertical pressure is increased, the radial pressure, which is able to keep the cavity with the same level of deformation, grows.

\section{The constitutive equations}

The shape of the response curves are mainly influenced by the constitutive equation in use. Next, the different models that have been used are analyzed.
Linear elastic model

It is present in all the following cases, at the beginning of the load, as well as in the unloading process. This model is a simple analytic solution, and can be used to verify the formulation of finite element models as well as their level of error.

\section{Perfect elastoplastic model}

A yield function has to be considered in this case. When the level of tension is higher than the function, plastic deformations appear.

The function of yielding used is static. There is no hardening. The plastic deformation is proportional to the gradient of a potential function, different from the yield function, that is to say, a model of non-associative plasticity is used.

Two functions of yielding are used. They are the MorhCoulomb's hexagonal pyramid and the Drucker-Prager's cone.

In the following figures the results obtained from both models for the same geotechnical parameters, kinds of response and initial state are shown.

The results are similar, although the conic model produces more rounded curves. The three main tensions are represented in each figure (Fig. 4a, b).

The Morh-Coulomb's perfect elastoplastic model without deformation and vertical pressure equal to $-v\left(\sigma_{\mathrm{r}}+\sigma_{\theta}\right)$ allows a verification with the finite elements model of the PLAXIS program (Brinkgreve 2004). The following graph shows the degree of adjustment reached (Fig. 5).

An additional result of this model is the spatial distribution of pressures in the inner soil. These values are presented in the following graph, for a fixed step size, as a function of the distance from the cavity (Fig. 6).

\section{Cam-Clay model}

Through this model two additional variables are introduced. First of all, it is a model whose yield function is frontally closed. Secondly, this frontal closure is a yield function with hardening.

The results of the model are shown in the figure below (Fig. 7). In order to have a criterion of comparison with the perfect elastoplastic models, the adjustment, previously obtained with Plaxis for Morh-Coulomb's model, is maintained.

This new model increases the number of variables with which a field test can be adjusted, among others, the initial consolidation pressure. The load way followed in the previous test is depicted in a p-q diagram (Fig. 8). 

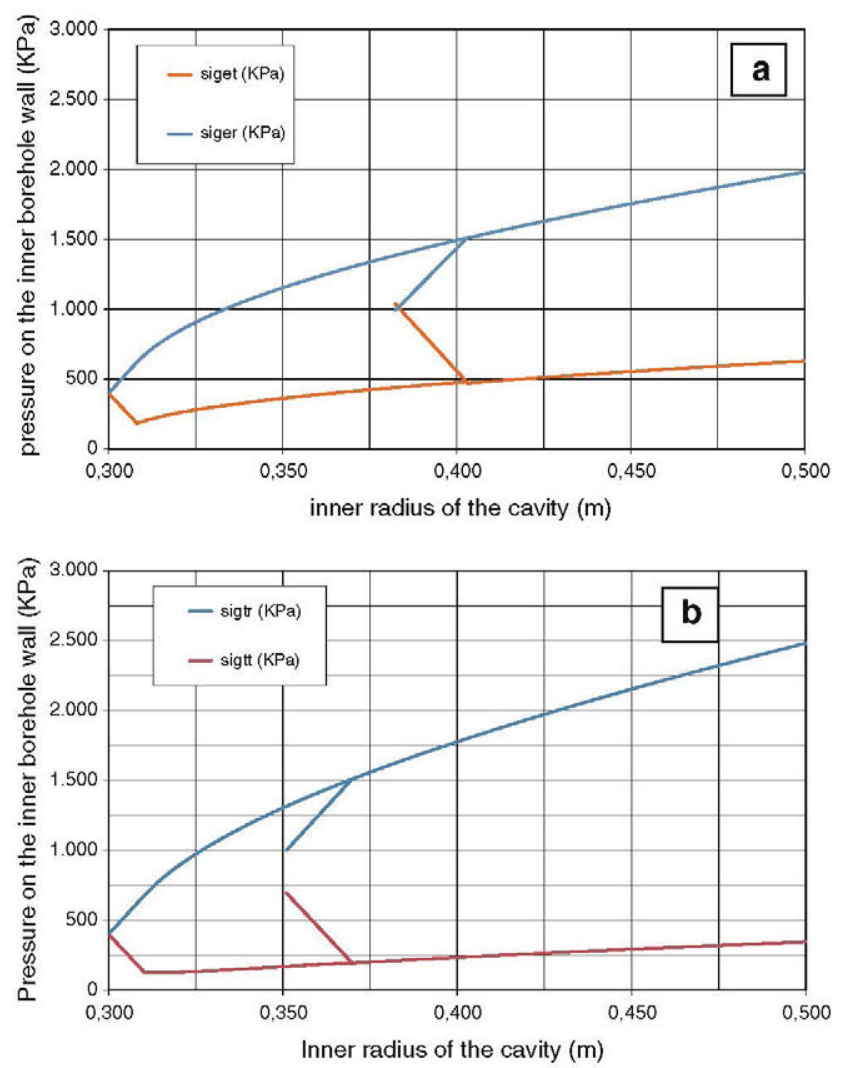

Fig. 4 a Morh-Coulomb's model. b Drucker-Prager's model

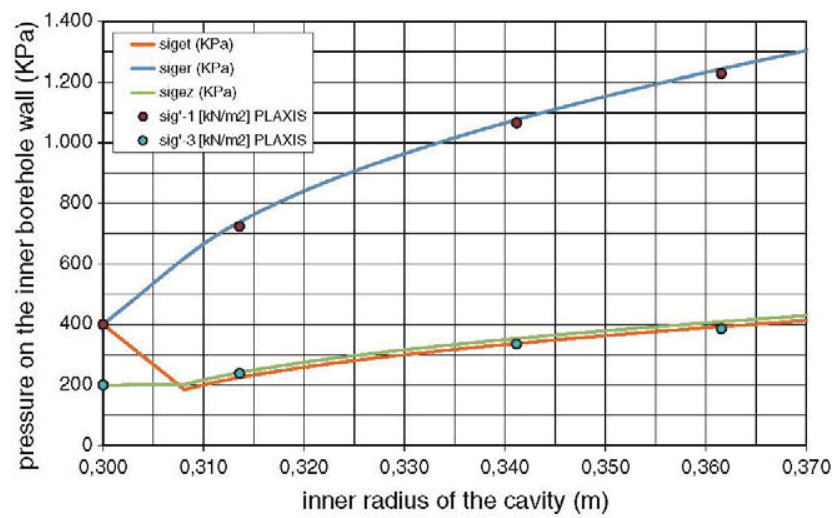

Fig. 5 Verification using PLAXIS program

The test begins in the elastic range, reaches the yield function of the frontal closure and makes it increase. Finally, the critical state is attained and the tests follow the breakage of the straight line provided by Morh-Coulomb's parameters.

\section{Other possible equations}

The numerical model depicted allows the use of other constitutive equations, like the Schanz's hardening soil

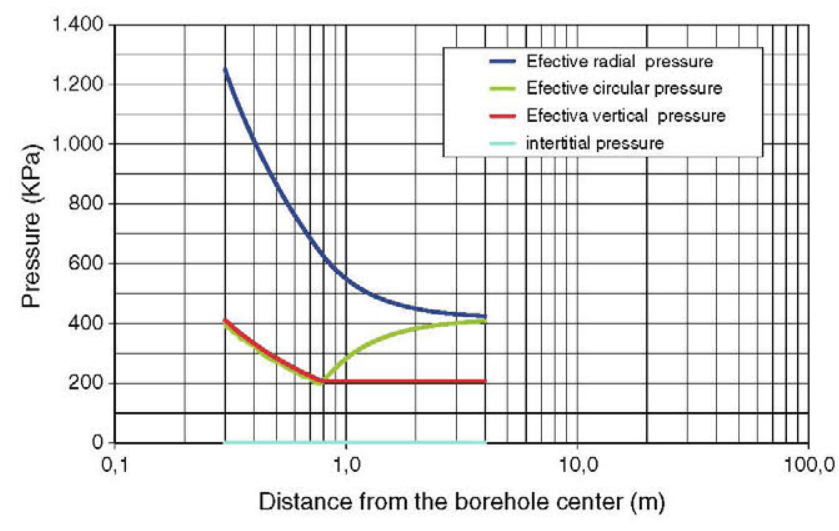

Fig. 6 Distribution of stresses inside soil

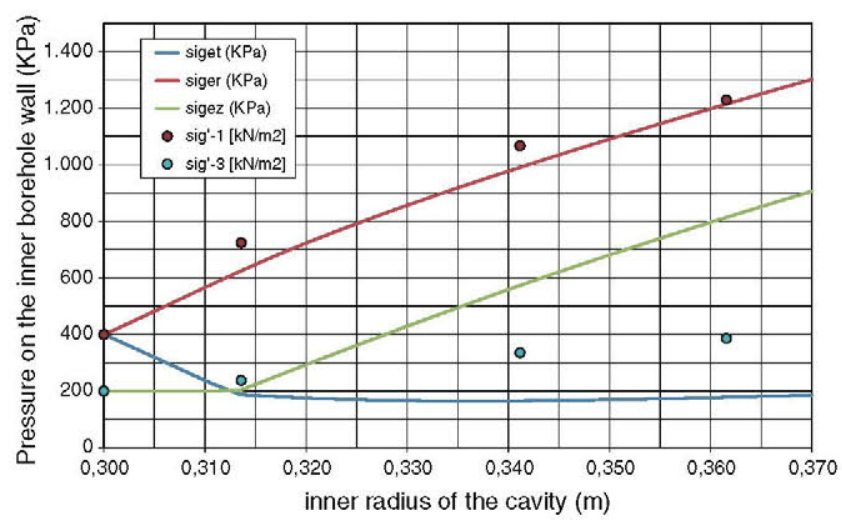

Fig. 7 Cam-Clay's model

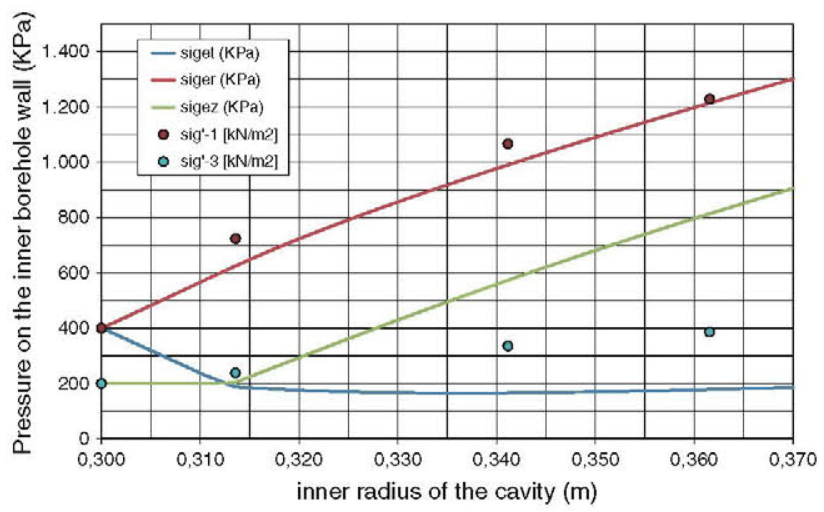

Fig. 8 Load way Cam-Clay's model

model (Vermeer 2000). This model has been used by the authors in some other papers.

However, most of the basic features of the new models that can be introduced are included in those previously explained. The analysis of the field tests curves according to the four referred models allow establishment of a reference framework. 
By means of this framework the behaviour of the soil surrounding a cavity, which is expanding, can be examined. The features which cannot be justified in the previous framework will be developed in new models.

\section{Undrained response}

The soil response can take place in drained conditions with dissipation of interstitial pressures or in undrained conditions (Aubeny et al. 2000; Benoit 1995).

In both cases the effective pressures are those that intervene in relation to the deformations through the constitutive equation. However, the drained response has two peculiarities:

1. The volumetric deformation of the solid skeleton is invalid.

2. The pressure measured inside the cavity is the sum of the pore and effective pressures.

The first condition is simulated with the numerical model eliminating the elastic and plastic deformation. The elastic deformation is suppressed by a Poisson coefficient next to 0.5 whereas the plastic deformation is removed differently in each model. A 0-dilatancy angle is used to erase perfect plastification models.

The interstitial pressure is generated by assigning a deformation module to the interstitial water, so there are two superimposed systems, water and soil, whose final volumetric deformation is equal and next to zero.

This modelling is depicted in the graph below (Fig. 9). The undrained models allow for justifying high pressures inside the cavity for low deformation levels and geotechnical parameters of soils having low cohesion.

\section{Adapted mesh}

The simplicity of the model used lets the calculation mesh be modified in each stage, so that the two distortional elements are eliminated; and it is possible to enter the states with large deformations.

In previous modelling, the hypothesis of small deformations was included and all the variables were referred to an initial mesh of fixed radius.

The modification of the mesh is carried out in each stage, defining a new family of radius whose inner edge is the one of the deformed meshes in the previous stage.

The tensions and deformations corresponding to an element "i" of the adapted mesh are modified to fulfill the equilibrium equations. The following figure depicts the results with two kinds of calculation (Fig. 10).

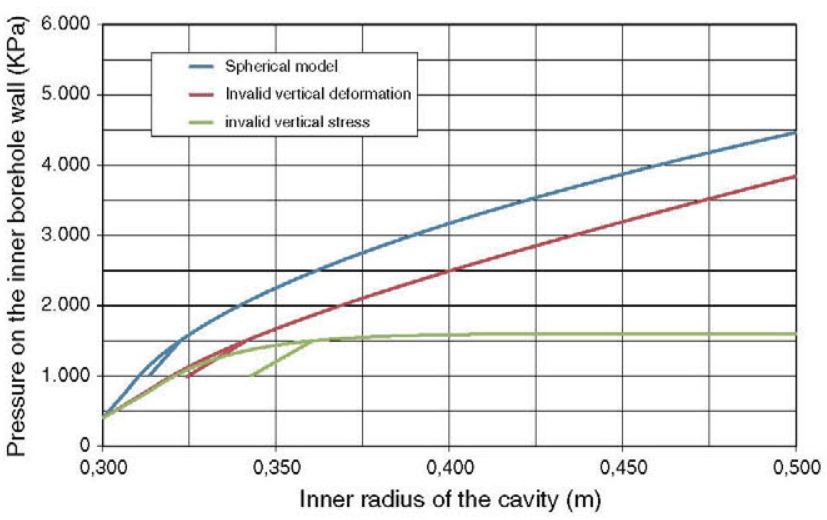

Fig. 9 Undrained model

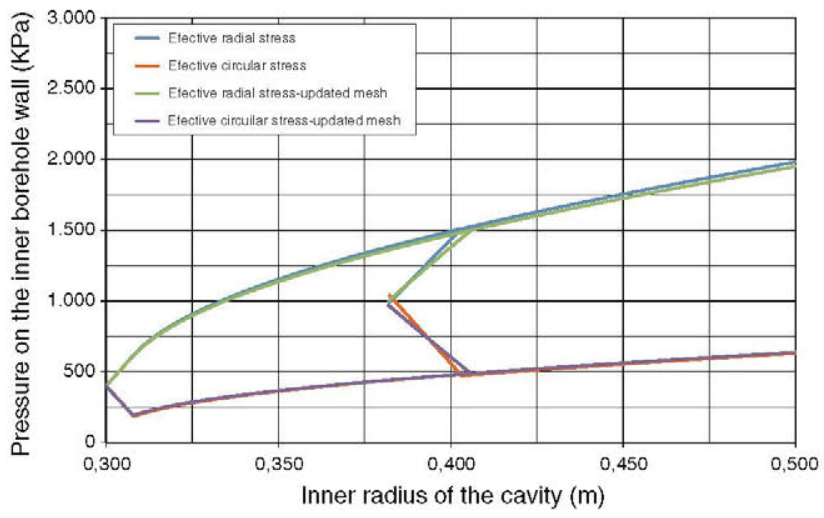

Fig. 10 Model with adapted mesh

It is observed how in considering a modelling that is more realistic (that is, the mesh adapting to the deformations), the results are not substantially modified.

\section{Conclusions}

The pressuremeter test is a load test, which involves several phenomena related to the tensor of deformation of the soil (Clough 1990).

This paper presents a reference framework based on the finite element numerical model; it is useful to compare the field test results with the expected soil response on the basis of its constitutive equation.

The geotechnical parameters are obtained defining an initial state, a kind of response and a constitutive equation of the soil.

The analyses carried out have determined the influence of the initial stress state, the kind of response (axisymmetric or spherical) and the constitutive equation.

A model has been proposed to analyze the cases of undrained behaviour. Finally, an improvement of the mathematical model is introduced to adapt it to deformations. 


\section{Future developments}

The generated reference framework provides a tool to analyze the field tests. Therefore, the use of the present model in a determined geological formation, whose tests are large and representative enough, implies a new method to diagnose geotechnically the unit (Dincer et al. 2008). In the future, the use of this reference framework in geotechnical surveys will show its potentiality.

With reference to the models described, several aspects have not been developed, among others, each model can be extended to a wider group of constitutive equations, specially the hardening soil model.

The consideration of an undrained behaviour close to the cavity and a drained one in farther locations, that is to say, a mixed response, may be possible.

The models with plastic flow have not been dealt with yet, although this parameter is measured in the pressuremeter tests in which the load is introduced in stages and plastic flow is measured in some of them.

Acknowledgments The authors of this paper wish to thank the company EUROCONSULT for the support provided in preparing the paper presented.

\section{References}

AENOR (1999) Geotecnia. Ensayos de campo y de laboratorio, Normas UNE, Madrid
Arrua PA, Aiassa G (2009) Horizonal load transference p-y curve for silty loess soils. EIA 4:131-143

Aubeny CP, Whittle AJ, Ladd CC (2000) Effects of disturbance on undrained strengths interpreted from pressuremeter tests. J Geotech Geoenviron Eng 126:1133-1144

Benoit J (1995) Advances in pressuremeter technology with specific reference to clays. In: The pressuremeter and its New Avenues, Proceedings of the 4 th international symposium on pressuremeters. Sherbrooke, Balkema, pp 125-139

Brinkgreve RBJ (2004) Refrencia manual PLAXIS versión 8.1. Delf University of Technology and PLAXIS, Delf

Clough GW (1990) The development of pressuremeter testing. In: Proceedings of the third international symposium on pressuremeters. Organised by British Geotechnical Society and held at Oxford University, p 25. American Society of Civil Engineers

Dincer I, Aarr A, Ural S (2008) Estimation of strength and deformation properties of quaternary caliche deposits. Bull Eng Geol Environ 67(3):353-366

Haberfield CM, Johnston IW (1990) A numerical model for pressuremeter testing in soft rock. Geotechnique 40:569-580

Morilla I (2012) Interpretación de los ensayos geotécnicos en suelos. Madrid, pp 401-421

Schanz T, Vermeer PA, Bonnier PG (1999) The hardening soil model: formulation and verification. In: Beyond 2000 in Computational Geotechnics - 10 Years of PLAXIS. Balkema, Rotterdam. ISBN $905809040 \mathrm{X}$

Yagiz S, Akyol E, Sen G (2008) Relationship between the standard penetration test and the pressuremeter test on sandy silty clays: a case of study from Denizli. Bull Eng Geol Environ 67:405-410 